\title{
Photoelectric peculiarities of electric photographic and holographic recording media with ionic dyes
}

\author{
N.A.Davidenko, A.A.Ishchenko* \\ T. Shevchenko University, 64, vul. Volodymyrska, 01033 Kyiv, Ukraine \\ *Institute of Organic Chemistry, NAS of Ukraine, 5, vul. Murmanska, 04094 Kyiv, Ukraine
}

\begin{abstract}
The features of formation and relaxation of metastable connected states of cation-radical of carbazole and negatively charged fragment of dye molecule were investigated in the films of poly-Nepoxypropilcarbazole doped with intraionic and cation dyes. In films with intraionic dye these states collapse at irradiation with light from the range of dye absorption, and in films with cation dye such states do not collapse under light action. Observed peculiarities are caused by structure features of the dyes.
\end{abstract}

Keywords: amorphous molecular semiconductors, electron-hole pairs, photoconductivity, ionic dyes.

Paper received 08.10.99; revised manuscript received 16.12.99; accepted for publication 17.12.99.

Homological series of cation polymethyne dyes [1] with different anions and of organic dying cations are considered as charge photogeneration centers in amorphous molecular semiconductors (AMS) based on carbazole-containing polymers. A comparative analysis of these AMS as to efficiency of photo- and thermogeneration of charge carriers in dependency on a chemical and energetic structure of dyes was carried out. The photoconductivity of the investigated AMS within the visible and IR parts of the light spectrum was found to be proportional to a ionization potential of the dye molecule and inversely proportional to a length of polymethyne chain of chromophores conjugating. Electric conductivity of the investigated AMS in strong electric fields is a sum of three components [2]. The first component of the electric conductivity is peculiar to the PEPC films without dopants. It corresponds to holes current and is determined by thermal field generation of holes from traps occupied in the result of holes thermogeneration from uncontrolled impurity centers. The second component is also peculiar to the films without dopants and corresponds to an ionic current. The third component of the electric conductivity is determined by the thermal generation of the charge carriers from the dye molecules. For the ionic dyes an activation energy of the third component of the electric conductivity depends on the temperature. It is determined by the depth of the traps of thermogenerated holes forming in PEPC near the ionic dye molecules [3]. It was shown that the magnitude of the investigated AMS films electric conductivity upon the dye change is mainly determined by a dye and polymer capability to a formation of the traps for the holes. The last circumstance is an evidence that the electric conductivity of the AMS films with dyes within the temperature range of electric photographic and holographic media utilization is determined by the thermofield generation of the captured holes, but not by the ionic conductivity.

The films of poly-N-epoxypropilcarbazole (PEPC) cation dyes have rather high photoconductivity [4]. It is more preferable to use intracation dyes in electrophoto-graphic and holographic media due to their photogeneration ability both as to positive charges (holes), and the negative ones (electrons). The purpose of the present work was to find out features of optical and photoelectric properties intrinsic for the PEPC films with intraionic dyes in comparison with the ionic ones.

Intraionic dve HISD

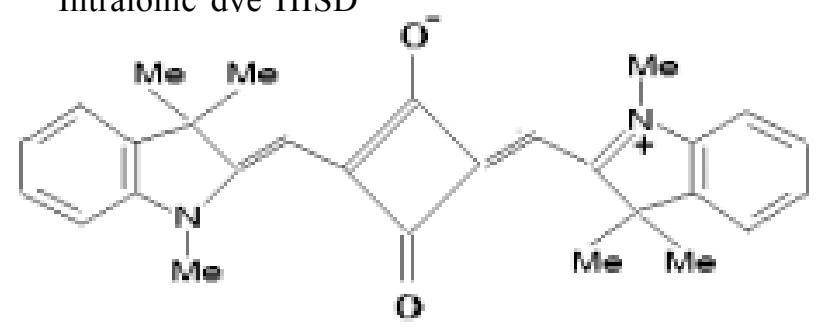

and ionic dye HIDC were used

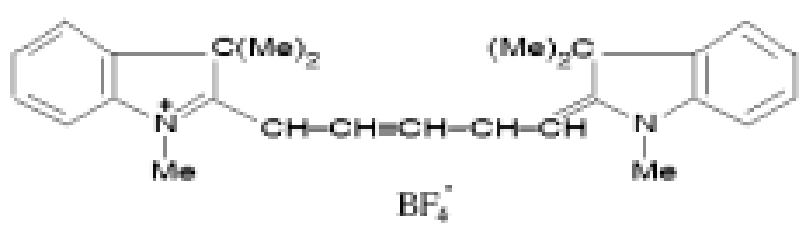

The excitation of a molecule of the first dye is accompanied by redistribution of electronic density from the negatively charged acceptor center $\left(\mathrm{A}^{-}\right)$to the positively charged donor chromophore $\left(\mathrm{D}^{+}\right)$. The excitation of a mol- 


\section{N.A.Davidenko, A.A.Ishchenko: Photoelectric peculiarities of electric photographic and...}

ecule of the second dye is accompanied by redistribution of electronic density from the less charged donor chromophore (D) to positively charged donor chromophore $\left(\mathrm{D}^{+}\right)$, but without electronic transfer from inorganic anion $\left(\mathrm{An}^{-}\right)$to cation.

The samples were prepared either as structures with a free surface: a quartz substrate-polymeric film, or as «sandwich» structures: Al-(polymeric film)- $\mathrm{SnO}_{2}$. The films PEPC+Nmas.\%HISD, PEPC+Nmas.\%HIDC for $N=1 \cdot 10^{-2}-5$ mas. $\%$ were used. The photocurrent $\left(j_{P H}\right)$ was measured in the samples of «sandwich» structure in a mode of photoresistance during illumination of the sample from the side of $\mathrm{SnO}_{2}$ electrode with wavelength $\lambda_{1}=380 \mathrm{~nm}$ (intensity $I_{1}$ ) or $\lambda_{2}=546,633,680$ and $711 \mathrm{~nm}$ (intensity $I_{2}$ ). Light intensities were $I_{1}=0.1-0.5 \mathrm{~W} / \mathrm{m}^{2}$ and $I_{2}=0.2-20 \mathrm{~W} / \mathrm{m}^{2}$. Chosen electric filed strength was $E=2 \cdot 10^{7}-3 \cdot 10^{8} \mathrm{~V} / \mathrm{m}$. In the samples with free surface optical spectra of absorption coefficient $(k)$ and photoluminescence intensity $\left(I_{P L}\right)$ were measured within the range of wavelengths $\lambda=350-1200 \mathrm{~nm}$. An optical densities $K_{1}$ and $K_{2}$ of the films for $\lambda_{1}$ и $\lambda_{2}$, respectively, was also determined. The values $\Delta K_{1}$ and $\Delta K_{2}$ being deviations of the films optical density from stationary value were calculated. Changes of $I_{P L}, K_{1}$ and $K_{2}$ as a result of pulse $(0.1 \mathrm{~s})$ heating the polymeric films up to temperatures $+(85-90){ }^{\circ} \mathrm{C}$ with the rate about $10^{6} \mathrm{~K} / \mathrm{s}$ were measured.

For creation of nonequilibrium concentration $(n)$ of the seized on a trap charges the samples were irradiated with light of $\lambda_{1}$ wavelength during time interval $t_{1}$. Chosen light wavelength is near the edges of PEPC absorption and is not absorbed by dye. Thus, there is a photo-generation and capture of mobile holes on traps created by carbazole nuclei (Cz) PEPC. Captured hole there corresponds to a cationradical $\left(\mathrm{Cz}^{+}\right)$with optical absorption in the visible part of the spectrum. Accumulation of $n$ was registered as reduction of $K_{1}$ and increase of $K_{2}$, and relaxation of $n$ could be observed due to restoration of $K_{1}$ and $K_{2}$ during the time interval $t_{2}$ after the irradiation.

In the samples Al-PEPC+Nmas.\%HISD-SnO 2 , after the beginning of irradiation with light, the photocurrent at first increases up to quasi stationary value, and after switching off the light decreases down to zero. But if after the irradiation with $\lambda_{1}$ light in time interval $t_{2}$ the sample is subjected to irradiation with $\lambda_{2}$ light, then on forward front of a current pulse the maximum is observed. Then photocur-rent decreases down to the quasi stationary value as it is schematically presented by diagrams in the insert of Fig.1. Using an amplitude of this maximum it is possible to estimate concentration $n$ of charges formed after the irradiation with $\lambda_{1}$ light and that taking part in photoconductivity that is stimulated by light with wavelength $\lambda_{2}$. The greatest influence of a preliminary irradiation with $\lambda_{1}$ light is observed at $\lambda_{2}=633 \mathrm{~nm}$, and it decreases up to zero when using $\lambda_{2}=711 \mathrm{~nm}$. It could be easily seen from the diagrams of dependencies $n\left(t_{1}\right)$ that it is possible to approximate these dependencies by idle time exponential function with time constant $3.1 \pm 0.2 \mathrm{~s}$ for $I_{2}=20 \mathrm{~W} / \mathrm{m}^{2}$. The value $n$ decreases with $t_{2}$ growth, is independent on $E$ in a time interval $t_{1}+t_{2}$, but decreases down to zero, if during the time interval $t_{2}$ even at $E=0$ the sample was illuminated with wavelength $\lambda_{2}$. The dependencies $n\left(t_{2}\right)$ can not be represented by the idle exponentially falling down function, but graphics of these ones could be approximated by linear dependencies in terms $n\left(\ln t_{2}\right)$. Tangent of a slope angle in these plots increases with $N$ (see Fig. 1). Dependencies of $\Delta K_{1}$ and $\Delta K_{2}$ on $\ln t_{2}$ correlate with each other as well as with dependency $n\left(t_{2}\right)$. The value $n$ decreases with an activation energy $0.17 \pm 0.03 \mathrm{eV}$ under $T$ growth within the temperature range of 293-370 K.

The magnitude of $I_{P L}$ decreases during the samples illumination. But if in time or after the irradiation the polymeric film is quickly heated up, the photoluminescence intensity is restored. Simultaneously with $I_{P L}$ restoration during the pulse heating we could observ restoration of $K_{1}$ and $K_{2}$.

In the samples Al-PEPC+Nmas. $\% \mathrm{HIDC}-\mathrm{SnO}_{2}$ being previously illuminated with $\lambda_{1}$ light the photocurrent maximum on forward front of kinetics is not observed upon switching on the light with wavelength $\lambda_{2}$. The dependencies $\Delta K_{1}$ and $\Delta K_{2}$ on $t_{1}$ could be presented by exponential function with time constant $25 \pm 5 \mathrm{~s}$, but the absolute values $\Delta K_{1}$ and $\Delta K_{2}$ are an order of magnitude less than that in the samples with the films PEPC+Nmas.\%HISD. The plots of $\Delta K_{1}$ and $\Delta K_{2}$ versus $t_{2}$ could be approximated by linear dependencies in terms $\Delta K_{1}$ and $\Delta K_{2}$ from $\ln t_{2}$ (see curve 4 in Fig. 1 ). The pulse heating of the polymeric films is accompanied by $I_{P L}$ reduction, and the $K_{1}$ and $K_{2}$ restoration was not revealed.

The results obtained testify that during the irradiation with wavelength $\lambda_{1}$ accumulation of nonequilibrium particles absorbing light in visible and near IR-range occurs. In the films PEPC+Nmas.\%HISD the rate of accumulation and relaxation of such particles is greater than in the films PEPC+Nmas.\%HIDC. Synchronously with the change of concentration of such particles the change of photoluminescence intensity of dyes happens. The distinction of the first kind of films from the second ones shows itself in different behavior: in the first ones, after an irradiation with wavelength $\lambda_{1}$ and following pulse heating $I_{P L}$ restores, and in the second ones it decreases. Besides, the excitation of HISD molecules results in acceleration of relaxation of these parti-

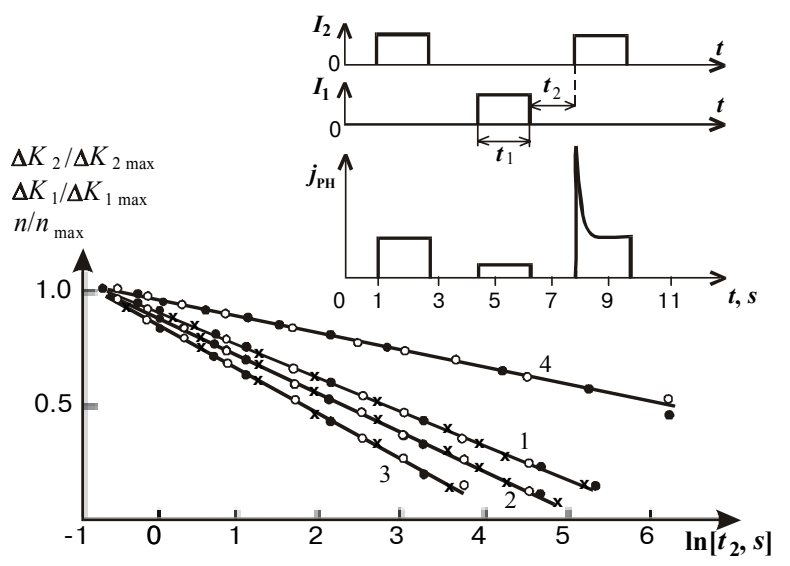

Fig.1. Dependencies of $\Delta K_{1}$ (dotes), $\Delta K_{2}$ (squares) and $n$ (crosses) on $\ln t_{2}$ for $t_{1}=1 \mathrm{c}, T=293 \mathrm{~K}, \lambda_{1}=380 \mathrm{~nm}, \lambda_{2}=633 \mathrm{~nm}$ in the samples with the films PEPC+Nmas.\%HISD (1-3) and PEPC+1mas.\%HIDC (4) for $N=0.01$ (1), 0.1 (2), 5 (3). 


\section{N.A.Davidenko, A.A.Ishchenko: Photoelectric peculiarities of electric photographic and...}

cles concentration, and the excitation of HIDC molecules does not render such action.

The results of measurements of the dependencies $n\left(t_{2}\right)$ specify that after irradiation with wavelength $\lambda_{1}$ some connected states appear in films. These ones consisting of positive and negatively charged particles, concentration reduction of which could be considered within the framework of recombination model of charge pairs with final spatial distribution on distances between these charges $[5,6]$. We propose the most adequate model based on the assumption of holes capture by $\mathrm{Cz}^{+}$near to charged fragments of dye molecules.

For intraionic dye this model can be presented by reactions:

1. After absorption in PEPC of light quantum $h v_{1}$ and dissociation of molecular exciton, the hole capture becomes possible near the negatively charged fragment of the dye, therefore the connected state $\left(\mathrm{Cz}^{+} \mathrm{A}^{-}\right)$appears.

2. The relaxation of the state $\left(\mathrm{Cz}^{+} \mathrm{A}^{-}\right)$has an activation character and is accompanied by the recombination of $\mathrm{Cz}^{+}$with electron staying after exciton dissociation.

3. The increase of $\mathrm{Cz}^{+}$concentration causes growth of the films absorption within the range of these cationradicals absorption and extinguishing of dye luminescence.

4. The absorption of light quantum with energy $h v_{2}$ by the dye molecule results in redistribution of electronic density in a molecule from $\mathrm{A}^{-}$to $\mathrm{D}^{+}$, that in turn is the reason of breaking connection between the charges in the state $\left(\mathrm{Cz}^{+} \mathrm{A}^{-}\right)$.

5. After liberation of $\mathrm{Cz}^{+}$charge, it becomes possible either its recombination, or creation of free hole $\left(p^{+}\right)$ participating in the induced photoconductivity.

For PEPC films with ionic dye HIDC the primary proc- esses of $\mathrm{Cz}^{+}$creation is the same, but the connected state is formed with a dye anion $\mathrm{An}^{-}$. At absorption of light quantum $\mathrm{hn}_{2}$ there is a redistribution of electronic density inside dye cation of, but in such way that the connection between $\mathrm{Cz}^{+}$and $\mathrm{An}^{-}$does not broken. Therefore, the excitation of dye molecules with photon energy $\mathrm{hn}_{2}$ is not accompanied by liberation of a charge from the state $\left(\mathrm{Cz}^{+} \mathrm{An}^{-}\right)$and induced photoconductivity is absent.

The proposed model of the nonequilibrium charge carriers capture mechanism when considering PEPC films with dyes possesses also practical interest because it demonstrates opportunities of photoconductivity increase in such films for their application as recording media.

\section{References}

1. A.A. Ishchenko, Structure and Spectral Luminescence Properties of Polymethine Dyes. Naukova Dumka, Kiev, (1994).

2. N.G Kuvshinsky., N.A Davidenko., V.M Komko. Physics of Amorphous Molecular Semiconductors. Kiev, Lybid', (1994).

3. N. Davidenko, A. Ischenko, A. Kadaschuk, N. Kuvshinsky, N. Ostapenko, Yu. Skryshevsky. Study of photoconductivity and luminescence of amorphous molecular semiconductors doped by polymethine dyes. // Fuctional Materials, 5(3), pp.353-355 (1998).

4. N. Davidenko, A. Ishchenko. Peculiarity of radiative recombination in amorphous molecular semiconductors doped with polymethine dyes. // Advanced Materials for Optics and Electronics, 8, pp.201-209 (1998).

5. N.A. Davidenko, N.G. Kuvshinsky. Peculiarities of generation and dissociation of electron-hole pairs in amorphous molecular semiconductors containing molecules of compounds with intramolecular charge transfer. // J. Inf. Rec. Mats., 21, pp.185-197 (1993).

6. N.A. Davidenko, A.K. Kadashchuk, N.G. Kuvshinsky, N.I. Ostapenko, N.V. Lukashenko. Photoconductivity features of amorphous molecular semiconductor films containing compounds with intramolecular charge transfer. The nature of capture centers. // J.Inf.Recording, 24, pp.327-338 (1996). 